\title{
174-nm Mode Spacing in Dual-Wavelength Semiconductor Laser Using Nonidentical InGaAsP Quantum Wells
}

\author{
Chi-Chia Huang, Chin-Hui Cheng, Yi-Shin Su, and Ching-Fuh Lin, Senior Member, IEEE
}

\begin{abstract}
A very wide tuning range of dual-wavelength semiconductor lasers with properly designed nonidentical InGaAsP quantum wells is reported. As the external cavity of reflected-type grating telescope configuration is well aligned, the dual-wavelength operation can be achieved with a record wavelength separation as large as $174 \mathrm{~nm}(25 \mathrm{THz})$. The wide separation of two wavelengths is possible due to a proper modification of the external-cavity configuration and reduced gain competition of laser modes.
\end{abstract}

Index Terms-Broad-band wavelength tuning, dual-wavelength operation, optical amplifier, quantum-well laser, semiconductor laser.

D UAL-wavelength laser systems have potential applications in many areas such as wavelength-division multiplexing (WDM) communication system, two-wavelength interferometry, laser spectroscopy, differential lidar, optical data processing, and so on. Numerous approaches have been used to achieve simultaneous two-wavelength lasing in both fiber and semiconductor lasers. In fiber lasers, Takahashi et al. [1] has used an array of doped fiber amplifiers as a multiple-wavelength source. Battiato et al. [2] has used a codoped fiber laser as a common gain medium in a linear cavity for dual-wavelength operation. In semiconductor lasers, array of lasers with physically separated gain media for each wavelength is a common approach for multiple-wavelength operation [3], [4]. Dual-wavelength operation with a single semiconductor gain medium has also been achieved by Lin et al. [5] and Hidaka et al. [6]. The use of a single gain medium shared by both wavelengths has the advantage of simplicity because no elaborate fabrication and packaging procedures are required [7].

Under dual-wavelength operation, some sort of mode competition will occur, especially when the mode spacing is small [5], [8]. However, when the mode spacing is large enough, mode competition is significantly reduced, increasing the possibility of two-mode oscillation. On the other hand, the difficulty for dual-wavelength operation at very large mode spacing is caused by the limited bandwidth of the gain medium and the diffi-

Manuscript received February 5, 2003; revised September 11, 2003.

C.-C. Huang, C.-H. Cheng and Y.-S. Su are with the Graduate Institute of Electro-Optical Engineering and Department of Electrical Engineering, National Taiwan University, Taipei, Taiwan, R.O.C.

C.-F. Lin is with the Graduate Institute of Electro-Optical Engineering and Department of Electrical Engineering. He is also with the Graduate Institute of Electronics Engineering, National Taiwan University, Taipei, Taiwan, R.O.C. (e-mail: cfling@cc.ee.niu.edu.tw).

Digital Object Identifier 10.1109/LPT.2003.821050

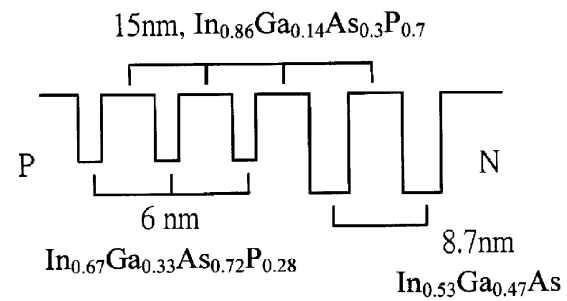

Fig. 1. QW structure of SOA used in the experiment.

culty of optical-path alignment in the external cavity. In this paper, we will demonstrate a mode spacing larger than $174 \mathrm{~nm}$ $(1356 \mathrm{~nm} \sim 1530 \mathrm{~nm})$ in dual-wavelength semiconductor lasers. The wide range of dual-wavelength operation indicates that the semiconductor optical amplifier (SOA) has the potential for operation at several wavelengths simultaneously. This could dramatically lower the cost and simplify the system when the SOA is used for WDM applications. Further increase of the mode spacing with the same device would be possible if the efficiency of the optical components in the external cavity was increased.

The dual-wavelength semiconductor laser uses a broad-band SOA as the gain medium. The SOA used in the experiment has nonidentical InGaAsP quantum-well (QW) structure, as shown in Fig. 1. This QW structure has very broad-band characteristics. The semiconductor laser using it as gain medium can be tuned from 1300 to $1540 \mathrm{~nm}$ under single-wavelength operation [9]. However, although the bent-stripe structure used in [9] provides the SOA with the ability of tuning at high injection current without the undesired Fabry-Perot resonance, this configuration has large internal loss. This is not good for dual-wavelength tuning where loss has a serious impact on the tuning range. Therefore, we use a straight-waveguide SOA in the experiment instead. No facet coating is applied. Although the straight waveguide will have undesired Fabry-Perot resonance, this phenomenon does not seriously affect the possibility of dual-wavelength operation with a large spacing. The reason will be discussed later. Fig. 2 shows the emission spectra of the SOA at different injection current. The threshold current of the Fabry-Perot resonance (at $1370 \mathrm{~nm}$ ) is about $143 \mathrm{~mA}$. In the experiment, the SOA is operated at 146-mA injection current and at temperature of $22.7^{\circ} \mathrm{C}$.

The experimental setup is shown in Fig. 3. The external cavity is similar to that described in [5] except that now the grating is of 600 lines $/ \mathrm{mm}$ and the lens is of $f=10 \mathrm{~cm}$. In addition, there are three elaborate arrangements in the external cavity. First, according to the grating principle, the beams of different 


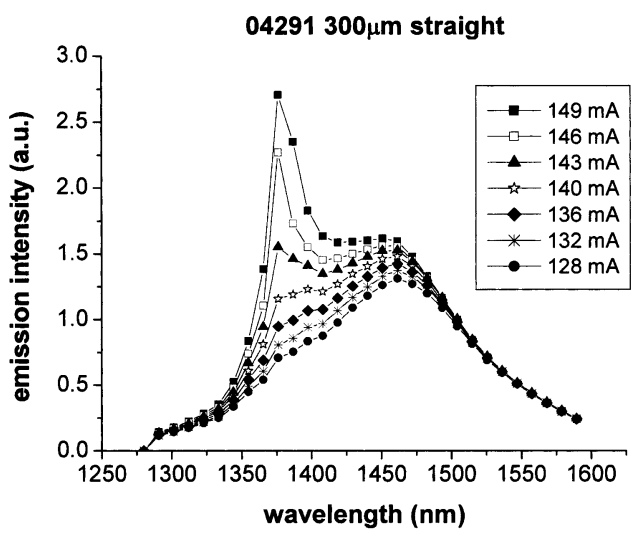

Fig. 2. Emission spectra of the SOA.

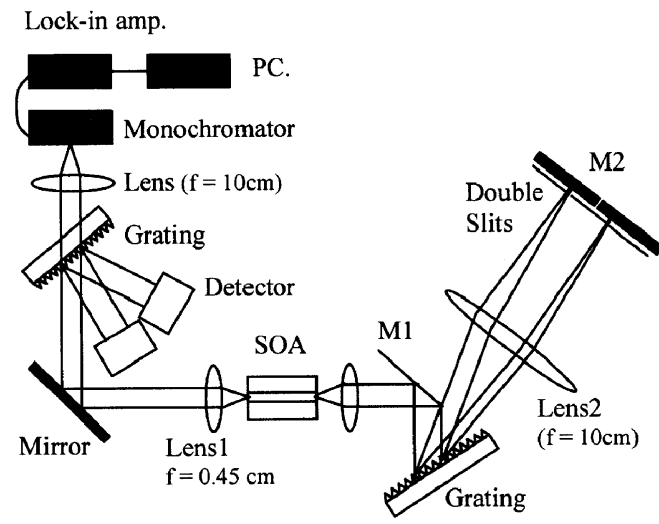

Fig. 3. Experimental setup.

wavelengths are horizontally dispersed at the mirror with a spatial separation $W \sim L(1 / \Lambda)\left(1 / \cos \theta_{R}\right) \Delta \lambda$, where $\Delta \lambda$ is the spectral separation, $\Lambda$ is the grating period, $\theta_{R}$ is the first-order diffraction angle, and $L$ is the distance between the lens and the grating. To obtain better diffraction efficiency, $\theta_{R}$ must be as small as possible. However, if $\theta_{R}$ is too small, the path of the input beam and the diffracted beam will be closely separated, and the holding stages of lens 2 and mirror M2 will block the path of the input beam. Therefore, we introduce an additional mirror M1 into the cavity and put the lens and M2 behind M1 to avoid blocking the input beam. To obtain minimum $\theta_{R}$, the light beam must be carefully aligned so that it passes just through the rim of mirror M1.

Second, at 174-nm mode spacing, the spatial separation of the two mode will be about $1.5 \mathrm{~cm}$. It means that the light will pass through a path away from the high efficiency center of lens2, which is of diameter $6 \mathrm{~cm}$. The loss in the short-wavelength mode can be compensated by increasing the injection current. However, at the same time the gain of the long-wavelength mode is reduced due to heating effect. Therefore, we make the longwavelength mode nearly pass through the high-efficiency center of lens2.

Third, as previously discussed, the spatial separation of the two modes is about $1.5 \mathrm{~cm}$. It is very hard to align M2 optimally for both wavelengths. Therefore, instead of using a single mirror for M2, we use two physically separated mirrors, so each of them can be aligned optimally for one wavelength. At the laser output, we use a grating to separate the light beam and

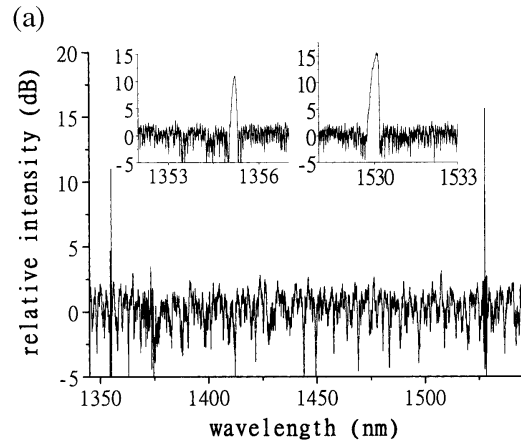

(b)

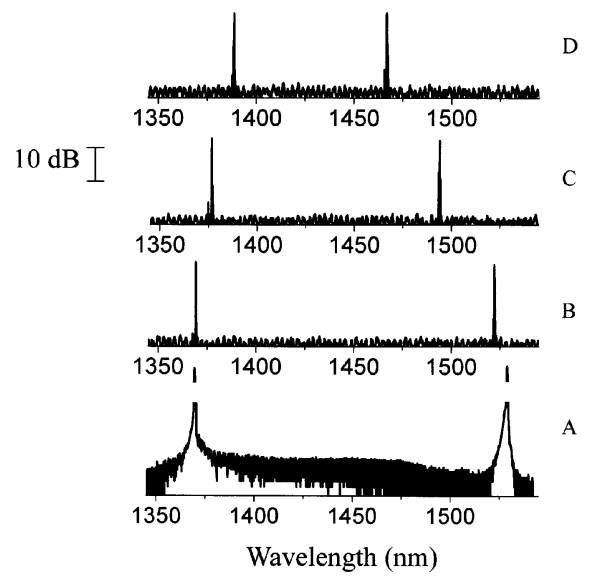

Fig. 4. Spectra for different spectral spacing: (a) spectral spacing $174.6 \mathrm{~nm}$ (1355.4 and $1530.1 \mathrm{~nm})$; (b) different spectral spacing: $A 159.1 \mathrm{~nm}(1371.0$ and $1530.1 \mathrm{~nm}), B 154.9 \mathrm{~nm}(1369.8$ and $1524.7 \mathrm{~nm}), C 118.2 \mathrm{~nm}(1377.4$ and $1495.6 \mathrm{~nm}), D 77.8 \mathrm{~nm}(1390.4$ and $1468.2 \mathrm{~nm})$.

use two detectors to measure the light power of each mode. Afterwards, we remove the grating and the detectors and use the monochromator to measure the spectrum.

Fig. 4(a) shows the maximum mode spacing. With shortwavelength mode at $1355.4 \mathrm{~nm}$ and long-wavelength mode at 1530.1 , the mode spacing is about $174.6 \mathrm{~nm}$, which corresponds to $25 \mathrm{THz}$ in frequency. This is the maximum mode spacing under which we can see two separate lasing peaks in the spectrum. Detailed spectrum for each mode is also shown in the figure. The FWHM of each lasing mode is less then $0.36 \mathrm{~nm}$. As the short-wavelength mode is shifted by $1.3 \mathrm{~nm}$ with respect to Fig. 4(a), the mode spacing is reduced to $173.3 \mathrm{~nm}$. Then the light intensity of the short-wavelength mode is nearly doubled, and the long-wavelength mode remains the same in terms of the wavelength and the intensity. The spectra for further shortening of the mode spacing are shown in Fig. 4(b), where $A, B, C$, and $D$ are spectra for four different mode spacings. They are plotted together for comparison. The full-width at half-maximum (FWHM) of each lasing mode is also less then $0.36 \mathrm{~nm}$. From Fig. 4, we can see that although the output power at 174.6-nm mode spacing is small, it could increase very fast with the reduction of the mode spacing until the mode spacing is about $130 \mathrm{~nm}$. Further reduction of the mode spacing does not increase the light power significantly. Light power of each mode at each mode spacing is listed in Table I. Although Fig. 4(b) only shows a narrow spacing of $77.8 \mathrm{~nm}$, the smallest mode spacing that can be tuned is less than $10 \mathrm{~nm}$. Moreover, at a fixed mode 
TABLE I

OUtPut Power of THE SHORT-WAVELENGTH Mode (PS) AND OUtPut Power of the Long-WaVelength Mode (PL) AT DifFerent Mode SPACING (SP)

\begin{tabular}{c|c|c}
\hline SP $(\mathrm{nm})$ & PS $(\mathrm{mW})$ & PL $(\mathrm{mW})$ \\
\hline 174.6 & 0.06 & 0.1 \\
\hline 173.3 & 0.12 & 0.19 \\
\hline 159.1 & 0.30 & 0.42 \\
\hline 154.9 & 0.51 & 0.41 \\
\hline 118.2 & 0.66 & 0.63 \\
\hline 77.8 & 0.6 & 0.62 \\
\hline
\end{tabular}

spacing, we can randomly choose the position of the two modes from 1355 to $1530 \mathrm{~nm}$ as long as the balance between the gain and the loss is properly adjusted. That is, there is no "gap" where dual-wavelength operation is forbidden.

In Fig. 4(a), the side-mode suppression ratio (SMSR) is about $10 \mathrm{~dB}$. The SMSR is larger than $20 \mathrm{~dB}$ for mode spacing less than $168 \mathrm{~nm}$. The measured spectra indicate that the power of amplified spontaneous emission (ASE) or spontaneous emission (SE) is quite low. Due to the instrumentation noise, the ASE level can not be observed in spectra $B, C, D$ of Fig. 4. However, when we increase the signal-to-noise ratio (SNR) of the measurement, we found that the SMSR is larger than $27 \mathrm{~dB}$, as shown in spectrum $A$ of Fig. 4. From the calculation based on spectrum $A$ of Fig. 4, 38\% of the total power belongs to the mode $1371.0 \mathrm{~nm}$, and $52 \%$ belongs to the mode $1530.1 \mathrm{~nm}$. Therefore, the power of ASE and SE are only $10 \%$ of the total power. The reason for the small ASE power is as follows. The broad-band SOA spreads out the spontaneous emission power to a very broad spectral range. This leads to the power of the lasing mode returned by the grating much larger than the power of other spectral range. Therefore, the lasing mode has much stronger gain-competition ability than light at other spectral range, so ASE noise is significantly suppressed.

The possibility of large mode spacing is due to the following reasons. First, the gain of the nonidentical QWs is broad [10]. Second, at large mode spacing, the two wavelengths are nearly contributed from two different QWs. The competition between them is weak because the carrier transportation between QWs is a relatively slow process, compared to the intraband relaxation in the same well. Moreover, during the experiment for Fig. 4(a), we found that the short-wavelength mode will offer the longwavelength mode some optical gain like optical pumping. This phenomenon suppresses mode competition completely. Instead, it behaves in a way opposite to competition.

The Fabry-Perot resonance has little impact on the broadening of the mode spacing when the injection current is not far above the threshold of the Fabry-Perot resonance. Our injection current is $146 \mathrm{~mA}$, which is larger than the threshold current of the Fabry-Perot mode by $3 \mathrm{~mA}$. However, experiments show that the Fabry-Perot mode could not oscillate when the grating-selected short-wavelength mode is lasing. Further broadening of the mode spacing would be possible with better alignment of the optical components in the external cavity. This could be achieved by adding some feedback control system to the holding stages of the optical components in the cavity instead of manual alignment.

In conclusion, using nonidentical InGaAsP QWs, the semiconductor laser could oscillate at two wavelengths with a very large spectral spacing. In a cavity formed between the output facet of the SOA and a reflection-type grating telescope, the mode spacing can be as large as $174 \mathrm{~nm}$. This large mode spacing is achieved due to the broad-band characteristics of the SOA and weaker competition of laser modes contributed from different QWs. Further broadening of the mode spacing is possible with better alignment of the optical components in the external cavity.

\section{REFERENCES}

[1] H. Takahashi, H. Toba, and Y. Inoue, "Multiwavelength ring laser composed of EDFA's and an arrayed-waveguide wavelength multiplexer," Electron. Lett., vol. 30, no. 1, pp. 44-45, 1994.

[2] J. M. Battiato, T. F. Morse, and R. K. Kostuk, "Dual-wavelength common-cavity codoped fiber laser," IEEE Photon. Technol. Lett., vol. 9, pp. 913-915, July 1997.

[3] K. O. Nyairo, I. H. White, C. J. Armistead, and P. A. Kirkby, "Multiple channel signal generation using multichannel grating cavity laser with crosstalk compensation," Electron. Lett., vol. 28, pp. 261-263, 1992.

[4] K. R. Poguntke, J. B. D. Soole, A. Scherer, H. P. LeBlance, C. Caneau, R. Baht, and M. A. Koza, "Simultaneous multiple wavelength operation of a multistripe array grating integrated cavity laser," Appl. Phys. Lett., vol. 62, pp. 2024-2026, 1993.

[5] C.-F. Lin, M.-J. Chen, and B.-L. Lee, "Wide-range tunable dual-wavelength semiconductor laser using asymmetric dual quantum wells," IEEE Photon. Technol. Lett., vol. 10, pp. 1208-1210, Sept. 1998.

[6] T. Hidaka and Y. Hatano, "Simultaneous two wave oscillation LD using biperiod binary grating," Electron. Lett., vol. 27, pp. 1075-1076, 1991.

[7] C. L. Wang and C. L. Pan, "Tunable dual-wavelength operation of a diode array with an external grating-loaded cavity," Appl. Phys. Lett., vol. 64, pp. 3089-3091, 1994.

[8] P.-C. Ku, C.-F. Lin, and B.-L. Lee, "Multiple cross switching in a two-mode semiconductor laser," Appl. Phys. Lett., vol. 69, pp. 3984-3986, 1996.

[9] C.-F. Lin, Y.-S. Su, and B.-R. Wu, "External-cavity semiconductor laser tunable from 1.3 to $1.54 \mu \mathrm{m}$ for optical communication," IEEE Photon. Technol. Lett., vol. 14, pp. 3-5, Jan. 2002.

[10] C.-F. Lin, B.-R. Wu, L.-W. Laih, and T.-T. Shih, "Sequence influence of nonidentical InGaAsP quantum wells on broadband characteristics of semiconductor optical amplifiers-superluminescent diodes," Opt. Lett., vol. 26, pp. 1099-1101, 2001. 